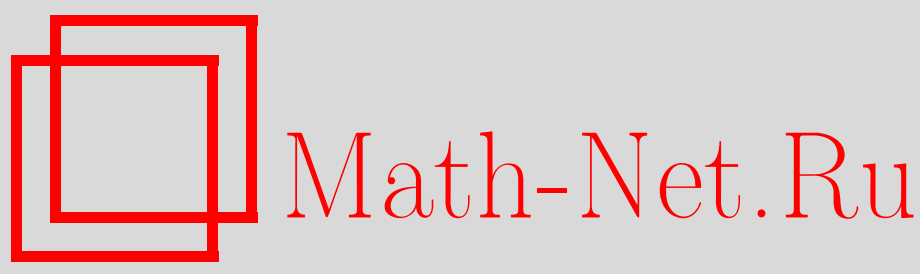

М. А. Королёв, Неполные суммы Клоостермана и их приложения, Изв. РАН. Сер. матем., 2000, том 64, выпуск 6, 41-64

DOI: https://doi.org/10.4213/im311

Использование Общероссийского математического портала Math-Net.Ru подразумевает, что вы прочитали и согласны с пользовательским соглашением

http://www . mathnet.ru/rus/agreement

Параметры загрузки:

IP : 3.93 .64 .190

26 апреля 2023 г., 16:51:03 
УДК 511

М. А. Королев

\section{Неполные суммы Клоостермана и их приложения}

Получена нетривиальная оценка верхней грани абсолютной величины неполной суммы Клоостермана, количество слагаемых в которой много меньше модуля.

Библиограффия: 11 наименований.

\section{Введение}

В теории чисел суммами Клоостермана называются тригонометрические суммы вида

$$
S_{1}=\sum_{n=1}^{m} \exp \left(2 \pi i \frac{a n^{*}+b n}{m}\right),
$$

где $m$ - натуральное число, $a, b$ - целые числа. Здесь и далее штрих означает суммирование по числам $n$, взаимно простым с $m$, а символом $n^{*}$ обозначено натуральное число, не превосходящее $m$ и такое, что $n^{*} n \equiv 1(\bmod m)$. Наряду с суммами (1) рассматриваются также суммы

$$
S_{2}=\sum_{n \leqslant x}^{\prime} \exp \left(2 \pi i \frac{a n^{*}+b n}{m}\right)
$$

где $x<m$, называемые неполными суммами Клоостермана.

Суммы (1) и (2) возникают при решении самых различных проблем теории чисел. Примерами служат задачи о количестве попаданий чисел вида $\left\{\frac{a n^{*}+b n}{m}\right\}$ в заданный интервал и об асимптотическом поведении суммы

$$
\sum_{n \leqslant x}^{\prime}\left\{\frac{a n^{*}+b n}{m}\right\} \text {. }
$$

Продвижение в этих задачах связано с получением нетривиальных оценок модулей сумм (1) и (2).

Первые такие оценки были получены в 1926 г. в [1]. Они имеют вид

$$
\begin{aligned}
& \left|S_{1}\right| \ll m^{\frac{3}{4}+\varepsilon} D^{\frac{1}{4}}, \\
& \left|S_{2}\right| \ll m^{\frac{7}{8}+\varepsilon} d^{\frac{1}{4}},
\end{aligned}
$$

где обозначено $d=(a, m), D=\max (d ;(b, m))$. 
В 1931 г. Г. Салье в статье [2] улучшил оценку (4) для случая $m=p^{\alpha}, p>2$ простое, $\alpha \geqslant 2,(a, p)=(b, p)=1$. Он получил

$$
\left|S_{1}\right| \leqslant 3 p^{\alpha / 2} \text {. }
$$

Из этого результата следует более общее неравенство

$$
\left|S_{1}\right| \leqslant \tau\left(p^{\alpha}\right) p^{\alpha / 2} d^{1 / 2} .
$$

Наконец, в 1948 г. появилась работа [3], в которой содержалось доказательство неравенства, подобного (6), для случая $m=p, p>2$ простое, $(a, p)=(b, p)=1$ :

$$
\left|S_{1}\right| \leqslant 2 p^{1 / 2} \text {. }
$$

С помошью известного свойства мультипликативности сумм Клоостермана и оценок $(7),(8)$ для произвольного нечетного модуля $m$ можно получить неравенство

$$
\left|S_{1}\right| \leqslant \tau(m) m^{\frac{1}{2}} d^{\frac{1}{2}} \ll m^{\frac{1}{2}+\varepsilon} d^{\frac{1}{2}} .
$$

Случай четного $m$ был изучен Карлитцом и Учиямой в [4], и для него были получены оценки, подобные (9).

Неравенство (9) позволяет оценить и неполную сумму:

$$
\left|S_{2}\right| \ll m^{\frac{1}{2}+\varepsilon} d^{\frac{1}{2}}
$$

Последний результат дает возможность получить асимптотику для суммы (3), начиная с $x \geqslant m^{\frac{1}{2}+\varepsilon}$, причем остаточный член в этой формуле имеет порядок $x^{\alpha}$, где $0<\alpha<1-$ некоторое число, не зависящее от $x$. Однако при $x<m^{1 / 2}$ в случае произвольного модуля $m$ к этой задаче до недавнего времени не было никаких подходов.

Метод, разработанный А. А. Карацубой (см. [5]-[9]), позволил получить нетривиальные оценки верхней границы модуля неполной суммы Клоостермана уже при $x \geqslant m^{\varepsilon}$, где $\varepsilon>0$ - сколь угодно малое фиксированное число. Более того, это удалось сделать и в ряде специальных случаев, когда параметр $n$ пробегает некоторое множество с количеством элементов $x \geqslant \exp \left(c(\lg m)^{3 / 4}(\lg \lg m)^{1 / 4}\right), c>0$ абсолютная постоянная. Это, в свою очередь, дало возможность получить асимптотическую формулу для суммы (3), уже начиная с $x \geqslant m^{\varepsilon}$. Соответствуюший результат А.А. Карацубы формулируется так.

ТЕОРемА. Пусть $\varepsilon-$ положительное сколь угодно малое фиксированное число, не превосходящее $0.001, x-$ вещественное число с условием $m^{\varepsilon} \leqslant$ $x \leqslant m, m x_{1}=\varphi(m) x, L=\lg m$. Тогда существует $c=c(\varepsilon)>0$ такое, что

$$
\sum_{n \leqslant x}^{\prime}\left\{\frac{a n^{*}+b n}{m}\right\}=\frac{1}{2} x_{1}\left(1+O\left(L^{-c}\right)\right) .
$$

(Сообщение об этом и некоторых других результатах см. в [7].)

В настоящей работе с помошью метода А.А. Карацубы и одного утверждения, являюшегося оригинальной частью работы (лемма А), доказывается, что асимптотическая формула для суммы (3) имеет место для любых $m$ при $x \geqslant$ $\exp \left(c \frac{\lg m}{\lg \lg m}\right), c>\lg 2$ - произвольная постоянная, а для некоторой бесконечной последовательности модулей (куда попадают, в частности, простые модули) - уже при $x \geqslant \exp \left((\lg m)^{4 / 5}(\lg \lg m)^{73 / 5}\right)$. 


\section{§1. Вспомогательные утверждения}

В этом параграфе будут приведены вспомогательные утверждения, необходимые для доказательства основной леммы и теорем.

ЛЕмма 1. При $x \geqslant 2$ имеет место равенство

$$
\sum_{p \leqslant x} \frac{1}{p}=\lg \lg x+c+\frac{\theta}{\lg x},
$$

где с $=0.26 \ldots-$-абсолютная постоянная, $a|\theta|<10$.

ЛЕмма 2. Пусть $a \geqslant 2, \quad b-a>1, \quad r \geqslant 0$ целое и $J$ - некоторое множество простых чисел из промежутка $(a, b]$. Пусть $w$ пробегает значения, являющиеся произведениями вида $w=p_{1} p_{2} \ldots p_{r}$, где $p_{i} \in J, i=1,2, \ldots, r, u$ $p_{1}<p_{2}<\cdots<p_{r}$. Тогда выполняется неравенство

$$
\sum_{w} \frac{1}{w} \leqslant \frac{1}{r !}\left(\sum_{p \in J} \frac{1}{p}\right)^{r}
$$

ДокАЗАТЕЛЬСТво. Пусть $q_{1}, \ldots, q_{s}$ - все простые числа из множества $J$. Если $s<r$, то утверждение леммы очевидно, так как в этом случае сумма в левой части неравенства равна нулю. Пусть $r \leqslant s$. Каждое из чисел $w$ может быть записано в виде $w=q_{1}^{\alpha_{1}} \ldots q_{s}^{\alpha_{s}}$, где $\alpha_{i}$ равно 0 или $1, i=1,2, \ldots, s$, и $\alpha_{1}+\cdots+\alpha_{s}=r$. При раскрытии скобок в правой части встретятся все слагаемые вида

$$
\frac{r !}{\alpha_{1} ! \ldots \alpha_{s} !}\left(q_{1}^{\alpha_{1}} \ldots q_{s}^{\alpha_{s}}\right)^{-1}=r ! w^{-1}
$$

Отсюда следует утверждение леммы.

Пусть $x \geqslant y \geqslant 2$. Обозначим $N(x, y)$ количество чисел ряда $1,2, \ldots,[x]$, все простые делители которых не превосходят $y$. Величину $\Phi(x, y)$ определим как количество чисел того же ряда, все простые делители которых больше $y$. Леммы 3 и 4 устанавливают верхние оценки для $N$ и $\Phi$, необходимые для дальнейшего.

Лемма 3. Пусть $y=y(x) \leqslant x$ и $y(x) \rightarrow+\infty$ при $x \rightarrow+\infty$. Тогда имеет место оченка

$$
N(x, y)<x \exp \left(-\lg x \frac{\lg \lg \lg y}{\lg y}+\lg \lg y+O\left(\frac{\lg \lg y}{\lg \lg \lg y}\right)\right),
$$

причем постоянная в знаке $О$ абсолютная.

Доказательство этой леммы см., например, в [10].

ЛЕмма 4. Пусть $x \geqslant y \geqslant y_{1}>1$. Тогда справедливо неравенство

$$
\Phi(x, y)<\frac{3 x \lg x}{(\lg y)^{2}} .
$$


ДокаЗАТЕльство. Разобьем рассматриваемые $\Phi(x, y)$ чисел на два класса. К первому отнесем бесквадратные числа, а ко второму - все остальные. Пусть $\Phi_{1}$ и $\Phi_{2}-$ количества чисел в первом и втором классах соответственно.

Возьмем целое $t \geqslant 0$. Обозначим $\Phi_{1}(t)$ количество чисел $n$ первого класса, представимых в виде $n=p_{1} \ldots p_{t+1}$, где $y<p_{1}<\cdots<p_{t+1} \leqslant x$. Тогда

$$
\begin{aligned}
\Phi_{1}(t) & =\sum_{p_{1} \ldots p_{t+1} \leqslant x} 1=\sum_{p_{1} \ldots p_{t} \leqslant x y^{-1}} \sum_{y<p_{t+1} \leqslant x\left(p_{1} \ldots p_{t}\right)^{-1}} 1 \\
& \leqslant \sum_{p_{1} \ldots p_{t} \leqslant x y^{-1}} \pi\left(\frac{x}{p_{1} \ldots p_{t}}\right) .
\end{aligned}
$$

При достаточно больших $h$ справедливо неравенство $\pi(h) \leqslant 2 h(\lg h)^{-1}$. Воспользовавшись этим неравенством и утверждениями лемм 1 и 2 , будем иметь

$$
\begin{aligned}
\Phi_{1}(t) & \leqslant 2 \sum_{p_{1} \ldots p_{t} \leqslant x y^{-1}} \frac{x}{p_{1} \ldots p_{t}}\left(\lg \frac{x}{p_{1} \ldots p_{t}}\right)^{-1} \\
& \leqslant 2 \frac{x}{\lg y} \sum_{p_{1} \ldots p_{t} \leqslant x y^{-1}} \frac{1}{p_{1} \ldots p_{t}} \leqslant 2 \frac{x}{\lg y} \cdot \frac{1}{t !}\left(\sum_{y<p \leqslant x} \frac{1}{p}\right)^{t} \\
& \leqslant 2 \frac{x}{\lg y} \cdot \frac{1}{t !}\left(\lg \lg x-\lg \lg y+\frac{20}{\lg y}\right)^{t} \\
& \leqslant 2 \frac{x}{\lg y} \cdot \frac{1}{t !}(\lg \lg x-\lg \lg y+0.1)^{t} .
\end{aligned}
$$

Так получаем оценку величины $\Phi_{1}$ :

$$
\Phi_{1}=\sum_{t=0}^{+\infty} \Phi_{1}(t) \leqslant 2 \frac{x}{\lg y} \sum_{t=0}^{+\infty} \frac{1}{t !}(\lg \lg x-\lg \lg y+0.1)^{t}=2 e^{0.1} \frac{x \lg x}{(\lg y)^{2}}
$$

Нетрудно видеть, что $\Phi_{2}$ не превосходит

$$
\sum_{l>y} \frac{x}{l^{2}}<\frac{2 x}{y}
$$

Таким образом,

$$
\Phi(x, y)=\Phi_{1}+\Phi_{2} \leqslant 2 e^{0.1} \frac{x \lg x}{(\lg y)^{2}}+\frac{2 x}{y}<\frac{3 x \lg x}{(\lg y)^{2}}
$$

Лемма доказана. 
Лемма 5 (А.А. Карацуба). Пусть $m>1$ - челое число, $k$ u $s$ - натуральные числа, вещественные числа $X, X_{1}, Y, Y_{1}$ удовлетворяют неравенствам

$$
\begin{aligned}
& k<X<X_{1}, \quad k X_{1}^{2 k-1}<m, \\
& s<Y<Y_{1}, \quad s Y_{1}^{2 s-1}<m,
\end{aligned}
$$

параметр $n$ пробегает $N$ значений натуральных чисел с условием $(n, m)=1$; a и $b$ - челье числа, причем $(a, m)=d ; p$ и q принимают значения последовательных простых чисел, причем $(p, m)=(q, m)=1$. Множество чисел вида прq, $X<p \leqslant X_{1}, \quad Y<q \leqslant Y_{1}$, обозначим буквой $A$, а количество әлементов А обозначим символом $\|A\|$. Тогда для тригонометрической суммы

$$
S=\sum_{n \in A} \exp \left(2 \pi i \frac{a n^{*}+b n}{m}\right)
$$

справедлива следующая оценка:

$$
|S| \leqslant\|A\| \Delta,
$$

¿əe

$$
\begin{gathered}
\Delta=(s ! k !)^{\frac{1}{2 s k}}\left(\pi_{1}(X)\right)^{-\frac{1}{2 s}}\left(\pi_{1}(Y)\right)^{-\frac{1}{2 k}}(s m d Y)^{\frac{1}{2 s k}}, \\
\pi_{1}(X)=\pi\left(X_{1}\right)-\pi(X)-\sum_{\substack{X<p \leqslant X_{1} \\
p \mid m}} 1 .
\end{gathered}
$$

Доказательство этого утверждения можно найти в [6].

ЛЕмма 6 ("стаканчик" Виноградова). Пусть $\rho$ - натуральное число, $\alpha u$ $\beta$ вещественные, $0<\delta<1 / 16,2 \delta<\beta-\alpha<1-2 \delta$. Тогда существует периодическая функиия $\psi(x)$ с периодом 1 и со следующими свойствами:

1) $\psi(x)=1$ при $\alpha+\delta \leqslant x \leqslant \beta-\delta$;

2) $0<\psi(x)<1$ nри $\alpha-\delta<x<\alpha+\delta, \beta-\delta<x<\beta+\delta$;

3) $\psi(x)=0$ nри $\beta+\delta \leqslant x \leqslant 1+\alpha-\delta$;

4) $\psi(x)$ разлагается в ряд Фурье вида

$$
\psi(x)=\beta-\alpha+\sum_{|r|>0} g(r) \exp (2 \pi i r x),
$$

zдe

$$
|g(r)| \leqslant \min \left(\beta-\alpha ; \frac{1}{\pi|r|} ; \frac{1}{\pi|r|}\left(\frac{\rho}{\pi|r| \delta}\right)^{\rho}\right)=c(r) .
$$


ЛЕмма 7. Пусть $\lambda_{1}, \lambda_{2}, \ldots, \lambda_{Q}$ - действительные числа с условием $0 \leqslant$ $\lambda_{s}<1, \quad s=1,2, \ldots, Q$. Пусть $\rho \geqslant 1$ иелое, $0<\delta<1 / 16, \alpha, \beta, R$ вещественные $, 2 \delta<\beta-\alpha<1-2 \delta, \psi(x)$ - функция из предыдущей леммы, отвечающая заданным $\rho, \delta, \alpha$ и $\beta$. Пусть при любых допустимых значениях $\alpha$ и $\beta$ для сумми

$$
U(\alpha, \beta)=\sum_{s=1}^{Q} \psi\left(\lambda_{s}\right)
$$

выполнено соотношение

$$
U(\alpha, \beta)=(\beta-\alpha) Q+O(R) .
$$

Тогда:

1) при любом $\sigma, 0 \leqslant \sigma<1$, число $A_{\sigma}$ значений $\lambda_{s}$ таких, что $0 \leqslant \lambda_{s}<\sigma$, выражается формулой

$$
A_{\sigma}=\sigma Q+R_{\sigma}, \quad R_{\sigma}=O(R)+O(Q \delta) ;
$$

2) $\sum_{s=1}^{Q} \lambda_{s}=\frac{1}{2} Q+O(R)+O(Q \delta)$.

Доказательство этого и предыдущего утверждений см., например, в [11].

\section{§2. Основная лемма}

Прежде чем сформулировать основное утверждение этого параграфа, введем ряд обозначений.

Пусть $m \geqslant m_{1}>1$ - натуральное число, $x$ - вешественное число, удовлетворяющее неравенствам

$$
\exp \left((\lg m)^{4 / 5}(\lg \lg m)^{5}\right)<x \leqslant m^{4 / 7},
$$

$C>1$ - абсолютная постоянная, точное значение которой будет выбрано позже. Положим также

$$
\begin{gathered}
U_{k}=\frac{1}{4} m^{\frac{1}{2 k-1}}, \quad V_{k}=m^{\frac{1}{2 k-1}-\frac{1}{4 k^{2}-1}}, \\
\gamma=\frac{5 \lg \lg x-4 \lg \lg m-\lg \lg \lg m-\lg C}{24 \lg \lg x}, \quad \varepsilon=\frac{\lg x}{\lg m}, \\
Q_{1}=\frac{1}{4} \exp \left(\frac{1}{4} \varepsilon(\lg x)^{1-10 \gamma}\right), \quad Q_{2}=\frac{1}{4} \exp \left(\frac{1}{4} \varepsilon(\lg x)^{1-8 \gamma}\right), \\
Q_{3}=\frac{1}{4} \exp \left((\lg x)^{1-4 \gamma}\right), \quad Q_{4}=\frac{1}{4} \exp \left((\lg x)^{1-2 \gamma}\right) .
\end{gathered}
$$

Нетрудно видеть, что

$$
\frac{\lg \lg \lg m}{2 \lg \lg m}<\gamma<\frac{5}{96}, \quad \frac{(\lg \lg m)^{5}}{(\lg m)^{1 / 5}}<\varepsilon \leqslant \frac{4}{7} .
$$

Целые числа $k_{1}, k_{2}, s_{1}$ и $s_{2}$, определяемые условиями

$$
\begin{aligned}
& U_{k_{1}} \leqslant Q_{4}<U_{k_{1}-1}, \quad U_{k_{2}+2}<Q_{3} \leqslant U_{k_{2}+1}, \\
& U_{s_{1}} \leqslant Q_{2}<U_{s_{1}-1}, \quad U_{s_{2}+1}<Q_{1} \leqslant U_{s_{2}}
\end{aligned}
$$


удовлетворяют неравенствам

$$
\begin{gathered}
\frac{1}{2 \varepsilon}(\lg x)^{2 \gamma}+\frac{1}{2} \leqslant k_{1}<\frac{1}{2 \varepsilon}(\lg x)^{2 \gamma}+\frac{3}{2} \\
\frac{1}{2 \varepsilon}(\lg x)^{4 \gamma}-\frac{3}{2}<k_{2} \leqslant \frac{1}{2 \varepsilon}(\lg x)^{4 \gamma}-\frac{1}{2} \\
\frac{2}{\varepsilon^{2}}(\lg x)^{8 \gamma}+\frac{1}{2} \leqslant s_{1}<\frac{2}{\varepsilon^{2}}(\lg x)^{8 \gamma}+\frac{3}{2} \\
\frac{2}{\varepsilon^{2}}(\lg x)^{10 \gamma}-\frac{5}{2}<s_{2} \leqslant \frac{2}{\varepsilon^{2}}(\lg x)^{10 \gamma}-\frac{3}{2}
\end{gathered}
$$

ЛЕмма А. Все натуральные числа $n$, не превосходящие $x$ и взаимно простые с $m$, за исключением не более чем

$$
\frac{60 x \lg \lg x}{(\lg x)^{\gamma}}
$$

чисел, можно представить в виде

$$
n=l^{2} p q h
$$

где l, h челье, $p, q$ простье, причем:

1) $\mu(p q h) \neq 0$

2) $1 \leqslant l \leqslant \lg x$

3) при некоторых $k$ и $s, k_{1} \leqslant k \leqslant k_{2}, s_{1} \leqslant s \leqslant s_{2}$, выполнены неравенства

$$
V_{k}<p \leqslant U_{k}, \quad U_{s+1}<q \leqslant U_{s} .
$$

ДокАЗАТЕЛЬСТво. Прежде всего изложим логическую схему доказательства. Станем отсеивать из ряда

$$
1,2,3, \ldots, n, \ldots,[x], \quad(n, m)=1
$$

те числа $n$, которые не могут быть представлены в виде (15). Процедура отсеивания состоит из нескольких шагов. На первом шаге (п. $\left.1^{\circ}\right)$ отбрасываются те $n$ из ряда (16), которые делятся на квадраты "больших" целых чисел. На втором шаге $\left(\right.$ п. $\left.2^{\circ}\right)$ специальным образом выбирается величина $Q=Q(x)$ и отбрасываются те числа, все простые делители которых больше $Q$, а также те числа, все простые делители которых не превосходят $Q$. Таким образом, все числа ряда $(16)$, оставшиеся после первых двух шагов, можно представить в виде $n=l^{2} n_{1}$, где $n_{1}$ - бесквадpaтное, a $l$ - маленькое целое число. При этом $n$ будет обладать по крайней мере одним простым делителем, не превосходящим $Q$, и хотя бы одним простым делителем, бо́льшим $Q$.

Последний, третий шаг (пп. $\left.3^{\circ}-5^{\circ}\right)$ является центральной частью доказательства. Здесь отсев происходит так. Сначала удаляются те $n$, у которых ни один простой делитель числа не попадает в промежуток $\left(U_{s_{2}+1} ; U_{s_{1}}\right]$. Тогда для каждого из оставшихся $n$ можно будет указать по крайней мере один простой делитель $q$, удовлетворяющий одному из неравенств $U_{s+1}<q \leqslant U_{s}, s=s_{1}, s_{1}+1, \ldots, s_{2}$. Наконец, 
из получившегося множества удаляем те $n$, у которых нет ни одного простого сомножителя из объединения промежутков $\left(V_{k} ; U_{k}\right], k=k_{1}, k_{1}+1, \ldots, k_{2}$. Нетрудно убедиться в том, что все числа ряда (16), оставшиеся после описанной процедуры, допускают представление в виде, указанном в формулировке леммы.

Отметим, что основную часть отбрасываемых чисел составляют удаляемые на последнем шаге. Станем доказывать утверждение, следуя изложенной схеме.

$1^{\circ}$. Количество чисел $n$ ряда (16), делящихся на квадрат "большого" целого числа $l>\lg x$, т.е. представимых в виде $n=l^{2} n_{1}, l>\lg x$, не превосходит величины

$$
\sum_{l>\lg x} \frac{x}{l^{2}}<\frac{2 x}{\lg x}
$$

$2^{\circ}$. Положим $Q=\exp \left(\frac{\lg x}{\lg \lg x}\right)$. Согласно леммам 3 и 4 количество чисел $n$ ряда (16), у которых либо все простые делители не превосходят $Q$, либо все простые делители больше $Q$, ограничено сверху величиной

$$
N(x, Q)+\Phi(x, Q)<\frac{4 x(\lg \lg x)^{2}}{\lg x} .
$$

Таким образом, отбросив

$$
<\frac{2 x}{\lg x}+\frac{4 x(\lg \lg x)^{2}}{\lg x}<\frac{5 x(\lg \lg x)^{2}}{\lg x}
$$

чисел, можно ограничиться рассмотрением тех $n \leqslant x$, которые удовлетворяют условиям $n=l^{2} n_{1}, 1 \leqslant l \leqslant \lg x, \mu\left(n_{1}\right) \neq 0$, причем $n$ делится на простое число, не превосходящее $Q$.

$3^{\circ}$. Для удобства записи введем следующие обозначения для промежутков:

$$
\begin{gathered}
J=\left(1 ; U_{s_{2}+1}\right], \quad J^{\prime}=\left(U_{s_{2}+1} ; U_{s_{1}}\right], \quad J^{\prime \prime}=\left(U_{s_{1}} ; Q\right], \quad J_{0}=\left(1 ; V_{k_{2}}\right] \\
J_{i}=\left(U_{k_{2}-i+1} ; V_{k_{2}-i}\right], \quad i=1,2, \ldots, \nu, \quad \nu=k_{2}-k_{1}, \\
J_{\nu+1}=\left(U_{k_{1}} ; Q\right] .
\end{gathered}
$$

Все дальнейшие рассуждения $\left(\right.$ п. $\left.4^{\circ}, 5^{\circ}\right)$ будут опираться на тот факт, что в каждом из этих промежутков, а также в каждом из промежутков $\left(V_{k} ; U_{k}\right], \quad k=$ $k_{1}, \ldots, k_{2}$, имеется достаточно много простых чисел, не делящих $m$. Покажем, что это действительно так.

Пусть $(u ; v]$ - любой из указанных промежутков. Покажем сначала, что

$$
\frac{u}{v} \leqslant \exp \left(-(\lg m)^{1 / 5}\right)
$$

Если $(u ; v]$ есть $J$ или $J_{0}$, то $u=1$,

$$
v \geqslant U_{s_{2}+1}=\frac{1}{4} m^{\frac{1}{2 s_{2}+1}} \quad \text { и } \quad \frac{u}{v} \leqslant m^{-\frac{1}{2 s_{2}+1}} .
$$


Если $(u ; v]$ есть $J^{\prime \prime}$ или $J_{\nu+1}$, то $v=Q, u \leqslant U_{k_{1}} \leqslant Q_{4}$, так что $u / v \leqslant Q_{4} / Q$. Наконец, в силу неравенств

$$
\begin{aligned}
U_{s_{2}+1} & <\frac{1}{4} m^{\frac{1}{2 s_{1}+1}}=U_{s_{1}} m^{-\frac{2}{4 s_{1}^{2}-1}}<U_{s_{1}} m^{-\frac{1}{4 s_{1}^{2}-1}}, \\
U_{k+1} & =\frac{1}{4} V_{k} m^{-\frac{1}{4 k^{2}-1}}<V_{k} m^{-\frac{1}{4 k_{2}^{2}-1}}<V_{k} m^{-\frac{1}{4 s_{1}^{2}-1}}, \quad k=k_{1}, \ldots, k_{2}-1, \\
V_{k} & =4 U_{k} m^{-\frac{1}{4 k^{2}-1}} \leqslant 4 U_{k} m^{-\frac{1}{4 k_{2}^{2}-1}}<4 U_{k} m^{-\frac{1}{4 s_{1}^{2}-1}}, \quad k=k_{1}, \ldots, k_{2},
\end{aligned}
$$

для каждого из оставшихся промежутков выполнено

$$
\frac{u}{v} \leqslant 4 m^{-\frac{1}{4 s_{1}^{2}-1}}
$$

Таким образом, достаточно доказать, что

$$
\max \left(m^{-\frac{1}{2 s_{2}+1}} ; Q_{4} Q^{-1} ; 4 m^{-\frac{1}{4 s_{1}^{2}-1}}\right)<\exp \left(-(\lg m)^{\frac{1}{5}}\right) .
$$

Воспользовавшись определением величин $\gamma$ и $\varepsilon$, а также неравенствами (13) и (14), получим

$$
\begin{aligned}
2 s_{2}+1 & <\frac{4}{\varepsilon^{2}}(\lg x)^{10 \gamma}=4 C^{-\frac{5}{12}}(\lg m)^{\frac{1}{3}}(\lg x)^{\frac{1}{12}}(\lg \lg m)^{-\frac{5}{12}} \\
& <4 C^{-\frac{5}{12}}(\lg m)^{\frac{1}{4}}(\lg \lg m)^{-\frac{5}{12}} \\
m^{-\frac{1}{2 s_{2}+1}} & <\exp \left(-\frac{C^{\frac{5}{12}}}{4}(\lg m)^{\frac{3}{4}}(\lg \lg m)^{\frac{5}{12}}\right)<\exp \left(-(\lg m)^{\frac{1}{5}}\right) \\
(\lg x)^{2 \gamma} & =C^{-\frac{1}{12}}(\lg \lg m)^{2}>2 \lg \lg x \\
Q_{4} Q^{-1} & =\frac{1}{4} \exp \left(-\frac{\lg x}{\lg \lg x}\left(1-\frac{\lg \lg x}{(\lg x)^{2 \gamma}}\right)\right) \\
& \leqslant \frac{1}{4} \exp \left(-\frac{\lg x}{2 \lg \lg x}\right)<\exp \left(-(\lg m)^{\frac{1}{5}}\right)
\end{aligned}
$$

наконец,

$$
\begin{gathered}
s_{1}<\frac{2}{\varepsilon^{2}}(\lg x)^{8 \gamma}+\frac{3}{2}, \quad 4 s_{1}^{2}-1<\frac{48}{\varepsilon^{4}}(\lg x)^{16 \gamma}=48 C^{-\frac{2}{3}}(\lg m)^{\frac{4}{5}}(\lg \lg m)^{-4} \\
4 m^{-\frac{1}{4 s_{1}^{2}-1}}<4 \exp \left(-\frac{C^{\frac{2}{3}}}{48}(\lg m)^{\frac{1}{5}}(\lg \lg m)^{4}\right)<\exp \left(-(\lg m)^{\frac{1}{5}}\right) .
\end{gathered}
$$

Далее, для каждого из рассматриваемых промежутков справедливо неравенство

$$
\begin{aligned}
v & \geqslant U_{s_{2}+1}=\frac{1}{4} m^{\frac{1}{2 s_{2}+1}}>\frac{1}{4} \exp \left(\frac{C^{\frac{5}{12}}}{4}(\lg m)^{\frac{3}{4}}(\lg \lg m)^{\frac{5}{12}}\right) \\
& >\exp \left((\lg m)^{\frac{3}{4}}(\lg \lg m)^{\frac{1}{3}}\right) .
\end{aligned}
$$


Поскольку при больших $h$

$$
\frac{1}{2} \cdot \frac{h}{\lg h} \leqslant \pi(h) \leqslant 2 \cdot \frac{h}{\lg h},
$$

то при $m \geqslant m_{1}$ имеем

$$
\begin{aligned}
\pi(v)-\pi(u) & \geqslant \frac{v}{2 \lg v}-\frac{2 u}{\lg u}=\frac{v}{2 \lg v}\left(1-4 \frac{u \lg v}{v \lg u}\right) \\
& >\frac{v}{2 \lg v}\left(1-4 \exp \left(-(\lg m)^{1 / 5}\right) \lg m\right)>\frac{v}{4 \lg v} \geqslant \frac{U_{s_{2}+1}}{4 \lg U_{s_{2}+1}} \\
& >\frac{\exp \left((\lg m)^{3 / 4}(\lg \lg m)^{1 / 3}\right)}{4(\lg m)^{3 / 4}(\lg \lg m)^{1 / 3}}>2 \exp \left((\lg m)^{3 / 4}\right) .
\end{aligned}
$$

Количество простых чисел промежутка $(u ; v]$, делящих $m$, не превосходит числа всех простых делителей $m$, которое, в свою очередь, не превосходит $2 \lg m$. Поэтому для числа простых чисел промежутка $(u ; v]$, взаимно простых с $m$, справедлива следуюшая оценка снизу:

$$
\pi(v)-\pi(u)-\sum_{\substack{u<p \leqslant v \\ p \mid m}} 1>2 \exp \left((\lg m)^{3 / 4}\right)-2 \lg m>\exp \left((\lg m)^{3 / 4}\right) .
$$

$4^{\circ}$. Пусть $N_{1}$ - количество чисел $n=l^{2} n_{1}$ ряда (16), оставшихся после первых двух шагов, которые не имеют простых делителей из промежутка $J^{\prime}$, и пусть $N_{1}(l)$ - количество таких $n$, отвечающих данному значению $l \leqslant \lg x$. Для каждого из этих $n$ число $n_{1}$ запишем в виде $n_{1}=w v$, где все простые делители $w$ не превосходят $Q$, а все простые делители $v$ больше $Q$. Поскольку в каноническом разложении $n$ отсутствуют простые числа из промежутка $J^{\prime}$, то все простые делители $w$ можно разбить на две группы. В первую войдут все делители $w$, принадлежашие промежутку $J$, а во вторую - принадлежашие промежутку $J^{\prime \prime}$. Поэтому число $w$ может быть представлено в виде

$$
w=p_{1} \ldots p_{r_{0}} q_{1} \ldots q_{r_{1}},
$$

где $r_{0} \geqslant 0, r_{1} \geqslant 0, p_{i} \in J, i=1,2, \ldots, r_{0}, q_{j} \in J^{\prime \prime}, j=1,2, \ldots, r_{1}$. Так как $n_{1}-$ бесквадратное число, то все простые $p_{1}, \ldots, p_{r_{0}}, q_{1}, \ldots, q_{r_{1}}$ различны.

Обозначим $N_{1}\left(l ; r_{0}, r_{1}\right)$ количество таких $n$, отвечаюших данным значениям $r_{0}$ и $r_{1}$. Для целого $r \geqslant 0$ положим

$$
N_{1}(l ; r)=\sum N_{1}\left(l ; r_{0}, r_{1}\right),
$$

где суммирование ведется по всем наборам $r_{0}, r_{1}$, для которых $r_{0}+r_{1}=r$. Зафиксируем произвольное число $w$ вида (17). Вспоминая определение величины $\Phi(x, y)$, получаем, что данному $w$ отвечает

$$
\Phi\left(\frac{x}{l^{2} w}, Q\right)
$$


чисел $v$ таких, что $n=l^{2} w v \leqslant x$. Фиксируем $r_{0}$ и $r_{1}$. Суммируя (18) по всем $w$, отвечающим выбранным $r_{0}$ и $r_{1}$, и пользуясь оценкой леммы 4 , получаем

$$
N_{1}\left(l ; r_{0}, r_{1}\right)=\sum_{w} \Phi\left(\frac{x}{l^{2} w}, Q\right)<\frac{3 x \lg x}{(l \cdot \lg Q)^{2}} \sum_{w} \frac{1}{w} .
$$

Для оценки последней суммы по $w$ используем неравенство леммы 2 :

$$
\sum_{w} \frac{1}{w} \leqslant \frac{1}{r_{0} !}\left(\sum_{p \in J} \frac{1}{p}\right)^{r_{0}} \cdot \frac{1}{r_{1} !}\left(\sum_{q \in J^{\prime \prime}} \frac{1}{q}\right)^{r_{1}} .
$$

Изучим отдельно каждую из сумм. Применяя лемму 1 , будем иметь

$$
\begin{gathered}
\sum_{p \in J} \frac{1}{p}=\sum_{\substack{2 \leqslant p \leqslant U_{s_{2}+1} \\
(p, m)=1}} \frac{1}{p} \leqslant \sum_{2 \leqslant p \leqslant U_{s_{2}+1}} \frac{1}{p} \leqslant \lg \lg U_{s_{2}+1}+c+\frac{10}{\lg U_{s_{2}+1}}, \\
\sum_{q \in J^{\prime \prime}} \frac{1}{q}=\sum_{\substack{U_{s_{1}}<q \leqslant Q \\
(q, m)=1}} \frac{1}{q} \leqslant \sum_{U_{s_{1}}<q \leqslant Q} \frac{1}{q} \leqslant \lg \lg Q-\lg \lg U_{s_{1}}+\frac{20}{\lg U_{s_{1}}} .
\end{gathered}
$$

С помошью неравенств (14) нетрудно получить

$$
\begin{aligned}
U_{s_{2}+1} & <\exp \left(\frac{1}{3} \varepsilon(\lg x)^{1-10 \gamma}\right), \\
U_{s_{1}} & >\exp \left(\frac{1}{5} \varepsilon(\lg x)^{1-8 \gamma}\right),
\end{aligned}
$$

как только $m \geqslant m_{1}$. Поэтому

$$
\begin{aligned}
& \lg \lg U_{s_{2}+1}+c+\frac{10}{\lg U_{s_{2}+1}} \leqslant \lg \lg U_{s_{2}+1}+\frac{1}{2} \\
& \leqslant(1-10 \gamma) \lg \lg x+\lg \varepsilon-\lg 3+\frac{1}{2}=\alpha_{0}, \\
& \lg \lg Q-\lg \lg U_{s_{1}}+\frac{20}{\lg U_{s_{1}}} \leqslant \lg \lg Q-\lg \lg U_{s_{1}}+\frac{1}{2} \\
& \quad \leqslant 8 \gamma \lg \lg x-\lg \lg \lg x-\lg \varepsilon+\lg 5+\frac{1}{2}=\alpha_{1} .
\end{aligned}
$$

Собрав полученные оценки вместе, последовательно находим

$$
\begin{gathered}
\sum_{w} \frac{1}{w} \leqslant \frac{\alpha_{0}^{r_{0}}}{r_{0} !} \cdot \frac{\alpha_{1}^{r_{1}}}{r_{1} !}, \\
N_{1}\left(l ; r_{0}, r_{1}\right)<\frac{3 x \lg x}{(l \cdot \lg Q)^{2}} \cdot \frac{\alpha_{0}^{r_{0}}}{r_{0} !} \cdot \frac{\alpha_{1}^{r_{1}}}{r_{1} !}, \\
N_{1}(l ; r)<\frac{3 x \lg x}{(l \cdot \lg Q)^{2}} \cdot \frac{1}{r !} \sum_{r_{0}+r_{1}=r} \frac{r !}{r_{0} ! r_{1} !} \alpha_{0}^{r_{0}} \alpha_{1}^{r_{1}}=\frac{3 x \lg x}{(l \cdot \lg Q)^{2}} \cdot \frac{\left(\alpha_{0}+\alpha_{1}\right)^{r}}{r !}, \\
N_{1}(l)=\sum_{r=0}^{+\infty} N_{1}(l ; r)<\frac{3 x \lg x}{(l \cdot \lg Q)^{2}} \sum_{r=0}^{+\infty} \frac{\left(\alpha_{0}+\alpha_{1}\right)^{r}}{r !}=\frac{3 x \lg x}{(l \cdot \lg Q)^{2}} \exp \left(\alpha_{0}+\alpha_{1}\right) .
\end{gathered}
$$


Так как

TO

$$
\alpha_{0}+\alpha_{1}=(1-2 \gamma) \lg \lg x-\lg \lg \lg x+\lg \left(\frac{5 e}{3}\right),
$$

$$
N_{1}(l)<\frac{3 x \lg x}{(l \cdot \lg Q)^{2}} \cdot \frac{(\lg x)^{1-2 \gamma}}{\lg \lg x} \cdot \frac{5 e}{3}=\frac{5 e x \lg \lg x}{l^{2}(\lg x)^{2 \gamma}} .
$$

Из равенства

$$
N_{1}=\sum_{l \leqslant \lg x} N_{1}(l)
$$

получаем верхнюю оценку величины $N_{1}$ :

$$
N_{1}<5 e \frac{x \lg \lg x}{(\lg x)^{2 \gamma}} \sum_{l \leqslant \lg x} \frac{1}{l^{2}}<\frac{5 \pi^{2} e}{6} \cdot \frac{x \lg \lg x}{(\lg x)^{2 \gamma}} .
$$

Исключим из рассматриваемого множества эти $N_{1}$ чисел. Тогда для каждого из оставшихся чисел $n$ можно указать хотя бы один простой делитель $q$ такой, что $(q, m)=1$ и $q \in J^{\prime}$. Поскольку

$$
J^{\prime}=\left(U_{s_{2}+1} ; U_{s_{1}}\right]=\bigcup_{s=s_{1}}^{s_{2}}\left(U_{s+1} ; U_{s}\right],
$$

то для такого $q$ можно единственным образом указать число $s$ так, что будут выполнены неравенства

$$
U_{s+1}<q \leqslant U_{s}, \quad s_{1} \leqslant s \leqslant s_{2} .
$$

$5^{\circ}$. Пусть $N_{2}$ - количество чисел $n=l^{2} n_{1}$ из множества, получившегося в п. $4^{\circ}$, которые не имеют простых делителей из объединения промежутков $\left(V_{k} ; U_{k}\right], k=$ $k_{1}, k_{1}+1, \ldots, k_{2}$, а $N_{2}(l)$ - количество таких $n$, которые отвечают данному значению $l \leqslant \lg x$. При оценке величин $N_{2}(l)$ и $N_{2}$ будем следовать рассуждениям п. $4^{\circ}$.

Для каждого из рассматриваемых чисел $n$ бесквадратный сомножитель $n_{1}$ запишем в виде $n_{1}=w v$, где все простые делители $w$ (если такие есть) попадают в объединение промежутков $J_{0}, J_{1}, \ldots, J_{\nu+1}$, а все простые делители $v$ больше $Q$. Для каждого $i, i=0,1, \ldots, \nu+1$, определим число $r_{i} \geqslant 0$ как количество простых делителей $w$, попавших в промежуток $J_{i}$. Обозначим $N_{2}\left(l ; r_{0}, r_{1}, \ldots, r_{\nu+1}\right)$ количество чисел $n$ из рассматриваемого множества, отвечающих данным значениям $l$, $r_{0}, r_{1}, \ldots, r_{\nu+1}$. Наконец, для целого $r \geqslant 0$ положим

$$
N_{2}(l ; r)=\sum N_{2}\left(l ; r_{0}, r_{1}, \ldots, r_{\nu+1}\right),
$$

где суммирование ведется по всем наборам $r_{0}, r_{1}, \ldots, r_{\nu+1}$, для которых $r_{0}+$ $r_{1}+\cdots+r_{\nu+1}=r$.

Рассуждая так же, как и в п. $4^{\circ}$, получим неравенство

$$
N_{2}\left(l ; r_{0}, r_{1}, \ldots, r_{\nu+1}\right)=\sum_{w} \Phi\left(\frac{x}{l^{2} w}, Q\right)<\frac{3 x \lg x}{(l \cdot \lg Q)^{2}} \sum_{w} \frac{1}{w},
$$

где суммирование проводится по всем числам $w$, которые отвечают данному набоpy $r_{0}, r_{1}, \ldots, r_{\nu+1}$. 
Применив лемму 2, оценим последнюю сумму по $w$ :

$$
\sum_{w} \frac{1}{w} \leqslant \prod_{i=0}^{\nu+1} \frac{\sigma_{i}^{r_{i}}}{r_{i} !}, \quad \sigma_{i}=\sum_{p \in J_{i}} \frac{1}{p}, \quad i=0,1, \ldots, \nu+1
$$

Займемся теперь величинами $\sigma_{i}$. Прежде всего, из неравенств $(14)$, которым удовлетворяет $k_{2}$, находим верхнюю оценку $V_{k_{2}}$ :

$$
V_{k_{2}}<\exp \left(2(\lg x)^{1-4 \gamma}\right)
$$

Воспользовавшись леммой 1, получим

$$
\begin{aligned}
\sigma_{0} & =\sum_{p \in J_{0}} \frac{1}{p} \leqslant \lg \lg V_{k_{2}}+c+\frac{10}{\lg V_{k_{2}}}<\lg \lg V_{k_{2}}+0.3 \\
& <(1-4 \gamma) \lg \lg x+\lg 2+0.3<(1-4 \gamma) \lg \lg x+1=\alpha_{0}
\end{aligned}
$$

Фиксируем $i, 1 \leqslant i \leqslant \nu$. Положим $k=k_{2}-i$. Тогда

$$
\sigma_{i}=\sum_{p \in J_{i}} \frac{1}{p}<\lg \lg V_{k}-\lg \lg U_{k+1}+\frac{20}{\lg U_{k+1}}=\lg \left(1+\frac{1}{2 k-1}\right)+\rho(k),
$$

где

$$
\rho(k)=\frac{20}{\lg U_{k+1}}-\lg \left(1-\frac{(2 k+1) \lg 4}{\lg m}\right) .
$$

Из (14) мы заключаем, что

$$
k \leqslant k_{2}<\frac{1}{10}(\lg m)^{1 / 3}-\frac{1}{2}
$$

Поэтому величина $\rho(k)$ по модулю не превосходит

$$
25 \cdot \frac{(2 k+1)}{\lg m}<\frac{1}{4(2 k-1)^{2}}
$$

Так, получаем

$$
\begin{aligned}
\sigma_{i} & <\lg \left(1+\frac{1}{2 k-1}\right)+\frac{1}{4(2 k-1)^{2}} \\
& <\frac{1}{2 k-1}-\frac{1}{4(2 k-1)^{2}}+\frac{1}{4(2 k-1)^{2}}=\frac{1}{2 k-1}=\frac{1}{2\left(k_{2}-i\right)-1}=\alpha_{i}
\end{aligned}
$$

Наконец, учитывая, что $U_{k_{1}}>\exp \left(\frac{1}{2}(\lg x)^{1-2 \gamma}\right)$, находим

$$
\begin{aligned}
\sigma_{\nu+1} & <\lg \lg Q-\lg \lg U_{k_{1}}+\frac{20}{\lg U_{k_{1}}} \\
& <\lg \lg Q-\lg \lg U_{k_{1}}+0.3<2 \gamma \lg \lg x-\lg \lg \lg x+1=\alpha_{\nu+1}
\end{aligned}
$$


Собирая полученные оценки вместе, получим серию неравенств:

$$
\begin{gathered}
\sum_{w} \frac{1}{w}<\prod_{i=0}^{\nu+1} \frac{\alpha_{i}^{r_{i}}}{r_{i} !} \\
N_{2}\left(l ; r_{0}, r_{1}, \ldots, r_{\nu+1}\right)<\frac{3 x \lg x}{(l \cdot \lg Q)^{2}} \prod_{i=0}^{\nu+1} \frac{\alpha_{i}^{r_{i}}}{r_{i} !}, \\
N_{2}(l ; r)<\frac{3 x \lg x}{(l \cdot \lg Q)^{2}} \sum_{r_{0}+r_{1}+\cdots+r_{\nu+1}=r} \frac{\alpha_{0}^{r_{0}} \alpha_{1}^{r_{1}} \ldots \alpha_{\nu+1}^{r_{\nu+1}}}{r_{0} ! r_{1} ! \ldots r_{\nu+1} !}=\frac{3 x \lg x}{(l \cdot \lg Q)^{2}} \cdot \frac{\alpha^{r}}{r !},
\end{gathered}
$$

где $\alpha=\alpha_{0}+\alpha_{1}+\cdots+\alpha_{\nu+1}$

$$
\begin{aligned}
N_{2}(l) & =\sum_{r=0}^{+\infty} N_{2}(l ; r)<\frac{3 x \lg x}{(l \cdot \lg Q)^{2}} \exp (\alpha), \\
N_{2} & =\sum_{l \leqslant \lg x} N_{2}(l)<\frac{\pi^{2}}{2} \cdot \frac{x \lg x}{(\lg Q)^{2}} \exp (\alpha) .
\end{aligned}
$$

Поскольку

$$
\begin{aligned}
\alpha & =(1-4 \gamma) \lg \lg x+1+2 \gamma \lg \lg x-\lg \lg \lg x+1+\sum_{k=k_{1}}^{k_{2}} \frac{1}{2 k-1} \\
& <(1-\gamma) \lg \lg x-\lg \lg \lg x+2,
\end{aligned}
$$

TO

$$
N_{2}<\frac{(\pi e)^{2} x \lg x}{2(\lg Q)^{2}} \cdot \frac{(\lg x)^{1-\gamma}}{\lg \lg x}=\frac{(\pi e)^{2} x \lg \lg x}{2(\lg x)^{\gamma}} .
$$

Исключим из рассмотрения и эти $N_{2}$ чисел. Таким образом, всего из ряда (16) оказалось исключено не более

$$
\frac{5 x(\lg \lg x)^{2}}{\lg x}+\frac{5 \pi^{2} e x \lg \lg x}{6(\lg x)^{2 \gamma}}+\frac{(\pi e)^{2} x \lg \lg x}{2(\lg x)^{\gamma}}<\frac{60 x \lg \lg x}{(\lg x)^{\gamma}}
$$

чисел. Для каждого из оставшихся можно указать хотя бы один простой делитель $p$, попавший в объединение промежутков $\left(V_{k} ; U_{k}\right], k=k_{1}, \ldots, k_{2}$. Наряду с (19) это означает, что все оставшиеся числа допускают представление (15). Лемма А доказана.

\section{§ 3. Оценка короткой суммы Клоостермана}

Пусть параметры $m, x, C, k_{1}, k_{2}, s_{1}, s_{2}, \gamma$ и $\varepsilon$ удовлетворяют условиям $\S 2, a, b$ целые числа, $(a, m)=d \geqslant 1$. В этом параграфе мы будем заниматься неполной суммой Клоостермана

$$
S=\sum_{n \leqslant x}^{\prime} \exp \left(2 \pi i \frac{a n^{*}+b n}{m}\right) .
$$


Теорема 1. Для суммы $S$ справедлива оценка

$$
|S| \leqslant x\left(\Delta_{1}+\Delta_{2}\right)
$$

əдe

$$
\begin{aligned}
& \Delta_{1}=d^{\frac{1}{2 k_{1} s_{1}}}(\lg m)^{-5}, \\
& \Delta_{2}=4(\lg x)^{-\frac{5}{24}}(\lg m)^{\frac{1}{6}}(\lg \lg m)^{\frac{25}{24}} .
\end{aligned}
$$

Доказательство. Разобьем сумму $S$ на две части: $S=S_{1}+S_{2}$. К сумме $S_{1}$ отнесем слагаемые, отвечающие тем $n$, которые имеют вид $n=l^{2} n_{1}, l \leqslant \lg x$, $\mu\left(n_{1}\right) \neq 0$. В $S_{2}$ войдут все остальные слагаемые. Нетрудно видеть, что число членов в $S_{2}$ не превосходит величины

$$
\sum_{\lg x<l \leqslant \sqrt{x}} \frac{x}{l^{2}}<\frac{2 x}{\lg x} .
$$

Оценивая $S_{2}$ тривиально, получим

$$
|S| \leqslant\left|S_{1}\right|+\frac{2 x}{\lg x} .
$$

Обозначим $A$ множество всех простых чисел, не деляших $m$ и содержашихся в объединении промежутков $\left(V_{k} ; U_{k}\right], k=k_{1}, \ldots, k_{2}$, а $B$ обозначим множество всех простых чисел, не делящих $m$ и содержашихся в промежутке $\left(U_{s_{2}+1} ; U_{s_{1}}\right]$.

Сумму $S_{1}$ разобьем на суммы $S_{\mu, \nu}$. K сумме $S_{\mu, \nu}$ отнесем все слагаемые, отвечающие числам $n$, которые могут быть представлены в виде $n=l^{2} h u=r u$, где $\mu(h u) \neq 0, a l \leqslant \lg x$. Здесь $u$ есть произведение $\mu$ простых сомножителей из $A$ и $\nu$ простых сомножителей из $B: u=p_{1} \ldots p_{\mu} q_{1} \ldots q_{\nu}$, a $r$ не имеет простых делителей из множеств $A$ и $B$.

Обозначим $\mu_{0}$ и $\nu_{0}$ максимальные возможные значения величин $\mu$ и $\nu$. Поскольку для любых $p \in A$ и $q \in B$ выполнены неравенства

$$
\begin{aligned}
& p>V_{k_{2}}>\exp \left((\lg x)^{1-4 \gamma}\right), \\
& q>U_{s_{2}+1}>\exp \left(\frac{1}{8} \varepsilon(\lg x)^{1-10 \gamma}\right),
\end{aligned}
$$

то для $\mu_{0}$ и $\nu_{0}$ получаем следующие оценки:

$$
\mu_{0} \leqslant(\lg x)^{4 \gamma}, \quad \nu_{0} \leqslant \frac{8}{\varepsilon}(\lg x)^{10 \gamma} .
$$

Те суммы $S_{\mu, \nu}$, где хотя бы один из индексов $\mu, \nu$ равен нулю, оценим тривиально. Слагаемые, входяшие в такие суммы, отвечают различным числам $n \leqslant x$, для которых невозможно указать пару простых делителей $p, q$ с условием: $p \in A$, $q \in B$. Согласно лемме А обшее число таких $n$ не превосходит величины $x \Delta$, где $\Delta=60 \lg \lg x /(\lg x)^{\gamma}$. Поэтому

$$
\left|S_{0,0}\right|+\sum_{\mu=1}^{\mu_{0}}\left|S_{\mu, 0}\right|+\sum_{\nu=1}^{\nu_{0}}\left|S_{0, \nu}\right| \leqslant x \Delta .
$$


Рассмотрим теперь сумму

$$
W=\sum_{r} \sum_{w \leqslant x r^{-1}} \exp \left(2 \pi i \frac{a n^{*}+b n}{m}\right), \quad n=r w .
$$

Здесь величина $r$ пробегает возрастающую последовательность чисел вида $r=$ $l^{2} h$, определенных выше. Величина $w$ принимает значения, являюшиеся произведениями $\mu+\nu$ простых сомножителей, каждый из которых независимо от других пробегает свою возрастающую последовательность чисел: $w=p_{1} \ldots p_{\mu} q_{1} \ldots q_{\nu}$. Именно, $p_{1}, \ldots, p_{\mu}$ независимо друг от друга пробегают множество $A$, a $q_{1}, \ldots, q_{\nu}-$ множество $B$.

Слагаемое, отвечающее значению $n=r w$, где $\mu(w) \neq 0$ (т.е. такому значению $w$, где все числа $p_{1}, \ldots, p_{\mu}, q_{1}, \ldots, q_{\nu}$ различны), встретится в сумме $W$ ровно $\mu ! \nu !$ раз.

Рассмотрим теперь слагаемое, отвечаюшее значению $n=r w$, где $\mu(w)=0$. Пусть среди чисел $p_{1}, \ldots, p_{\mu}$ оказалось ровно $g$ различных, причем первое встречается $\alpha_{1}$ раз, второе $-\alpha_{2}$ раз, $\ldots, g$-е $-\alpha_{g}$ раз. Пусть также среди чисел $q_{1}, \ldots, q_{\nu}$ ровно $t$ различных, причем первое повторяется $\beta_{1}$ раз, второе $-\beta_{2}$ раз, $\ldots, t$-е $\beta_{t}$ раз. Тогда $\alpha_{1}+\alpha_{2}+\cdots+\alpha_{g}=\mu$ и $\beta_{1}+\beta_{2}+\cdots+\beta_{t}=\nu$. Так $\operatorname{kak} \mu(w)=0$, то хотя бы одно из чисел $\alpha_{1}, \alpha_{2}, \ldots, \alpha_{g}, \beta_{1}, \beta_{2}, \ldots, \beta_{t}$ строго больше 1 . Поэтому соответствующее слагаемое встретится в сумме $W$

$$
\frac{\left(\alpha_{1}+\cdots+\alpha_{g}\right) !\left(\beta_{1}+\cdots+\beta_{t}\right) !}{\alpha_{1} ! \ldots \alpha_{g} ! \beta_{1} ! \ldots \beta_{t} !}<\mu ! \nu !
$$

раз. Заметим, что все такие $n=r w$ имеют вид: $n=l^{2} n_{1}, \mu\left(n_{1}\right) \neq 0, l>\lg x$. Поэтому количество таких чисел $n \leqslant x$ не превосходит величины

$$
\sum_{\lg x<l \leqslant \sqrt{x}} \frac{x}{l^{2}}<\frac{2 x}{\lg x} .
$$

Так, получаем

$$
\begin{gathered}
(\mu ! \nu !)^{-1} W=S_{\mu, \nu}+2 \theta \frac{x}{\lg x}, \quad|\theta|<1, \\
\left|S_{\mu, \nu}\right|<(\mu ! \nu !)^{-1}|W|+\frac{2 x}{\lg x} .
\end{gathered}
$$

Займемся оценкой суммы $W$. Обозначим $u=r p_{2} \ldots p_{\mu} q_{2} \ldots q_{\nu}, p=p_{1}, q=q_{1}$, $n=u p q$. Перепишем $W$ в виде

$$
W=\sum_{u} \sum_{\substack{p \in A \\ p q \leqslant x u^{-1}}} \sum_{\substack{q \in B \\ p}} \exp \left(2 \pi i \frac{a n^{*}+b n}{m}\right) .
$$

Множества $A$ и $B$, в которых меняются $p$ и $q$, разобьем на промежутки следующим образом. Каждый из сегментов $\left(V_{k} ; U_{k}\right], k=k_{1}, \ldots, k_{2}$, разобьем на промежутки

$$
\left(X ; X_{1}\right]=\left(\max \left(V_{k} ; 2^{-(\tau+1)} U_{k}\right) ; 2^{-\tau} U_{k}\right], \quad \tau=0,1, \ldots, \tau_{0} .
$$


Если для промежутка, отвечаюшего значению $\tau=\tau_{0}$, оказалось $X_{1}<2 X$, то объединим его с $\left(2^{-\tau_{0}} U_{k} ; 2^{-\left(\tau_{0}-1\right)} U_{k}\right]$. В итоге получим промежуток $\left(X^{\prime} ; X_{1}^{\prime}\right]$, для которого $2 X^{\prime} \leqslant X_{1}^{\prime} \leqslant 4 X^{\prime}$. Таким образом, для каждого получившегося промежутка $\left(X ; X_{1}\right]$ окажутся выполненными неравенства

$$
2 X \leqslant X_{1} \leqslant 4 X \text {. }
$$

Подобным образом каждый из промежутков $\left(U_{s+1} ; U_{s}\right], s=s_{1}, \ldots, s_{2}$, разобьем на промежутки $\left(Y ; Y_{1}\right]$, где $2 Y \leqslant Y_{1} \leqslant 4 Y$. Число получившихся при таком разбиении пар $X, Y$ не превосходит величины

$$
k_{2} s_{2} \log _{2}\left(U_{k_{1}}\right) \log _{2}\left(U_{s_{1}}\right)<(\lg m)^{3} .
$$

В соответствии с этим сумма $W$ разобьется на $<(\lg m)^{3}$ сумм

$$
W(X, Y)=\sum_{u \leqslant x(X Y)^{-1}} \sum_{\substack{X<p \leqslant X_{1} \\ X Y<p q \leqslant Z}}^{\prime} \sum_{\substack{Y<Y_{1} \\ Y}}^{\prime} \exp \left(2 \pi i \frac{a n^{*}+b n}{m}\right),
$$

где $Z=\left[\min \left(X_{1} Y_{1}, x u^{-1}\right)\right]$. Обозначим символом $W_{1}$ внутреннюю сумму по $p$ и $q$. Функцию $\delta(z)$ определим следуюшим образом:

$$
\delta(z)= \begin{cases}1, & z=p q, \quad(z, m)=1, \quad X<p \leqslant X_{1}, \quad Y<q \leqslant Y_{1}, \\ 0 & \text { в противном случае. }\end{cases}
$$

Тогда $W_{1}$ перепишется в виде

$$
W_{1}=\sum_{X Y<z \leqslant Z} \delta(z) \exp \left(2 \pi i \frac{a_{1} z^{*}+b_{1} z}{m}\right),
$$

где $a_{1}=a u^{*}, b_{1}=b u$. При этом $\left(a_{1}, m\right)=(a, m)=d$. Теперь преобразуем $W_{1}$ так, чтобы интервал изменения $z$ не зависел от $u$ :

$$
\begin{aligned}
W_{1}= & \sum_{X Y<z \leqslant X_{1} Y_{1}}\left(\frac{1}{m} \sum_{f=0}^{m-1} \sum_{y=1}^{Z} \exp \left(2 \pi i \frac{f(z-y)}{m}\right)\right) \delta(z) \exp \left(2 \pi i \frac{a_{1} z^{*}+b_{1} z}{m}\right) \\
=\frac{Z}{m} & \sum_{X Y<z \leqslant X_{1} Y_{1}} \delta(z) \exp \left(2 \pi i \frac{a_{1} z^{*}+b_{1} z}{m}\right) \\
+ & \frac{1}{m} \sum_{f=1}^{m-1} \frac{\exp \left(-2 \pi i \frac{f(Z+1)}{m}\right)-\exp \left(-2 \pi i \frac{f}{m}\right)}{\exp \left(-2 \pi i \frac{f}{m}\right)-1} \\
& \times \sum_{X Y<z \leqslant X_{1} Y_{1}} \delta(z) \exp \left(2 \pi i \frac{a_{1} z^{*}+\left(b_{1}+f\right) z}{m}\right) .
\end{aligned}
$$

Пусть $T$ - наибольшее возможное значение модуля суммы

$$
\sum_{X Y<z \leqslant X_{1} Y_{1}} \delta(z) \exp \left(2 \pi i \frac{a_{2} z^{*}+b_{2} z}{m}\right)=\sum_{X<p \leqslant X_{1}}^{\prime} \sum_{Y<q \leqslant Y_{1}}^{\prime} \exp \left(2 \pi i \frac{a_{2} p^{*} q^{*}+b_{2} p q}{m}\right),
$$


где $a_{2}, b_{2}$ - целые числа, $\left(a_{2}, m\right)=d$. Тогда, как нетрудно видеть,

$$
\left|W_{1}\right| \leqslant\left(\frac{Z}{m}+\frac{1}{m} \sum_{f=1}^{m-1}\left(\sin \frac{\pi f}{m}\right)^{-1}\right) T \leqslant\left(\frac{Z}{m}+\frac{1}{m} \sum_{f=1}^{m-1}\left(\frac{f}{m}\right)^{-1}\right) T<2 T \lg m .
$$

Для заданных $X, X_{1}, Y, Y_{1}$ определим $k$ и $s$ так, чтобы выполнялись неравенства

$$
\begin{gathered}
V_{k}=m^{\frac{1}{2 k-1}-\frac{1}{4 k^{2}-1}}<X<X_{1} \leqslant U_{k}=\frac{1}{4} m^{\frac{1}{2 k-1}}, \\
U_{s+1}=\frac{1}{4} m^{\frac{1}{2 s+1}}<Y<Y_{1} \leqslant U_{s}=\frac{1}{4} m^{\frac{1}{2 s-1}}, \\
k_{1} \leqslant k \leqslant k_{2}, \quad s_{1} \leqslant s \leqslant s_{2} .
\end{gathered}
$$

Тогда окажутся выполненными следующие условия:

$$
\begin{gathered}
k X_{1}^{2 k-1}<m, \quad s Y_{1}^{2 s-1}<m, \\
X>k \geqslant 3, \quad Y>s \geqslant 3 .
\end{gathered}
$$

Это позволяет нам применить лемму 5 . Так, получим

$$
T \leqslant X Y \Delta^{\prime}
$$

где

$$
\Delta^{\prime}=(k ! s !)^{\frac{1}{2 k s}}\left(\pi_{1}(X)\right)^{-\frac{1}{2 s}}\left(\pi_{1}(Y)\right)^{-\frac{1}{2 k}}(s m d Y)^{\frac{1}{2 k s}},
$$

a

$$
\pi_{1}(X)=\pi\left(X_{1}\right)-\pi(X)-\sum_{\substack{X<p \leqslant X_{1} \\ p \mid m}} 1 .
$$

Воспользовавшись известньм неравенством

$$
n !<e \sqrt{n}\left(\frac{n}{e}\right)^{n}
$$

а также тем, что $s>k \geqslant 3$, будем иметь

$$
\begin{aligned}
(k ! s !)^{\frac{1}{2 k s}} \cdot s^{\frac{1}{2 k s}} & \leqslant\left(e^{2} \sqrt{k s}\left(\frac{k}{e}\right)^{k}\left(\frac{s}{e}\right)^{s}\right)^{\frac{1}{2 k s}} s^{\frac{1}{2 k s}} \\
& =e^{-\left(\frac{1}{2 k}+\frac{1}{2 s}-\frac{1}{k s}\right)}(k s)^{\frac{1}{4 k s}} s^{\frac{1}{2 k s}} k^{\frac{1}{2 s}} s^{\frac{1}{2 k}} \\
& <(3 \cdot 3)^{\frac{1}{4 \cdot 3 \cdot 3}} \cdot 3^{\frac{1}{2 \cdot 3 \cdot 3}} \cdot 3^{\frac{1}{2 \cdot 3}} \cdot s^{\frac{1}{2 k}}<3^{\frac{1}{3}} s^{\frac{1}{2 k}} .
\end{aligned}
$$

Согласно неравенствам (14)

$$
\begin{aligned}
s^{\frac{1}{2 k}} & \leqslant s_{2}^{\frac{1}{2 k_{1}}} \leqslant \exp \left(\frac{\lg \left(2 \varepsilon^{-2}(\lg x)^{10 \gamma}\right)}{\varepsilon^{-1}(\lg x)^{2 \gamma}}\right)<\exp \left(\frac{(10 \gamma \lg \lg x-2 \lg \varepsilon+\lg 2)}{(\lg x)^{2 \gamma}}\right) \\
& \leqslant 2 \exp \left(\frac{\frac{25}{12} \lg \lg x-\frac{5}{3} \lg \lg m-2 \lg \lg x+2 \lg \lg m}{C^{-\frac{1}{12}}(\lg \lg m)^{2}}\right) \\
& =2 \exp \left(\frac{\frac{1}{12} \lg \lg x+\frac{1}{3} \lg \lg m}{C^{-\frac{1}{12}}(\lg \lg m)^{2}}\right) \leqslant 2 \exp \left(\frac{C^{\frac{1}{12}}}{4}(\lg \lg m)^{-1}\right)<3
\end{aligned}
$$


при $m \geqslant m_{1}$. Таким образом,

$$
(k ! s !)^{\frac{1}{2 k s}} s^{\frac{1}{2 k s}}<3^{\frac{4}{3}} .
$$

Далее, поскольку $X_{1} \geqslant 2 X$, для $\pi_{1}(X)$ справедлива оценка снизу:

$$
\pi_{1}(X) \geqslant \pi(2 X)-\pi(X)-2 \lg m
$$

Так как

$$
\pi(2 X)=\frac{2 X}{\lg X}+O\left(\frac{X}{(\lg X)^{2}}\right), \quad \pi(X)=\frac{X}{\lg X}+O\left(\frac{X}{(\lg X)^{2}}\right),
$$

то при достаточно больших $X$ (что достигается при $\left.m \geqslant m_{1}\right)$

$$
\pi_{1}(X) \geqslant \frac{X}{\lg X}+O\left(\frac{X}{(\lg X)^{2}}\right)-2 \lg m, \quad \pi_{1}(X) \geqslant \frac{X}{2 \lg X} .
$$

Аналогично, при $m \geqslant m_{1}$

$$
\pi_{1}(Y) \geqslant \frac{Y}{2 \lg Y}
$$

Поэтому

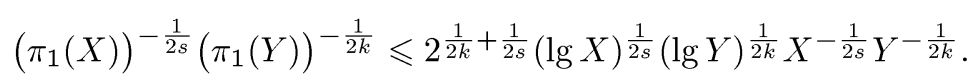

Так как $k \geqslant k_{1} \geqslant 3, s \geqslant s_{1}>3$, то

$$
\begin{gathered}
2^{\frac{1}{2 k}+\frac{1}{2 s}} \leqslant 2^{\frac{1}{6}+\frac{1}{6}}=2^{\frac{1}{3}}, \\
(\lg X)^{\frac{1}{2 s}} \leqslant \exp \left(\frac{\lg \lg m}{2 s_{1}}\right) \leqslant \exp \left(\frac{\lg \lg m}{\varepsilon^{-2}(\lg x)^{8 \gamma}}\right) \\
\leqslant \exp \left(\frac{\lg \lg m}{(\lg x)^{2 \gamma}}\right)=\exp \left(C^{\frac{1}{12}}(\lg \lg m)^{-1}\right) \leqslant 2, \\
(\lg Y)^{\frac{1}{2 k}} \leqslant \exp \left(\frac{\lg \lg m}{2 k_{1}}\right) \leqslant \exp \left(\frac{\lg \lg m}{\varepsilon^{-1}(\lg x)^{2 \gamma}}\right) \leqslant \exp \left(\frac{\lg \lg m}{(\lg x)^{2 \gamma}}\right) \leqslant 2,
\end{gathered}
$$

и поэтому

$$
\left(\pi_{1}(X)\right)^{-\frac{1}{2 s}}\left(\pi_{1}(Y)\right)^{-\frac{1}{2 k}} \leqslant 2^{\frac{7}{3}} X^{-\frac{1}{2 s}} Y^{-\frac{1}{2 k}}
$$

Таким образом,

$$
\Delta^{\prime} \leqslant 2^{\frac{7}{3}} 3^{\frac{4}{3}} d^{\frac{1}{2 k s}} X^{-\frac{1}{2 s}} Y^{-\frac{1}{2 k}+\frac{1}{2 k s}} m^{\frac{1}{2 k s}} .
$$

Принимая во внимание неравенства (21) и (22), будем иметь

$$
\begin{aligned}
& m^{\frac{1}{2 k s}} X^{-\frac{1}{2 s}} Y^{-\frac{1}{2 k}+\frac{1}{2 k s}} \\
& \quad \leqslant m^{\frac{1}{2 k s}}\left(m^{\frac{1}{2 k-1}-\frac{1}{4 k^{2}-1}}\right)^{-\frac{1}{2 s}}\left(\frac{1}{4} m^{\frac{1}{2 s+1}}\right)^{-\frac{1}{2 k}+\frac{1}{2 k s}} \leqslant 4^{\frac{1}{2 k}-\frac{1}{2 k s}} m^{-\delta},
\end{aligned}
$$


где

$$
\delta=\frac{s-6 k^{2}}{2 k s(2 s+1)\left(4 k^{2}-1\right)} .
$$

Обращаясь к (14), находим нижнюю границу для величины $\delta$ :

$$
\delta \geqslant \frac{\varepsilon^{5}}{16(\lg x)^{24 \gamma}}=\frac{C}{16} \frac{\lg \lg m}{\lg m} .
$$

Собрав все полученные оценки вместе, найдем верхнюю гранищу для $\Delta^{\prime}$ в виде

$$
\Delta^{\prime} \leqslant 2^{\frac{7}{3}} 3^{\frac{4}{3}} 4^{\frac{1}{6}} d^{\frac{1}{2 k s}}(\lg m)^{-\frac{C}{16}}<28 d^{\frac{1}{2 k s}}(\lg m)^{-\frac{C}{16}} .
$$

Наконец, замечая, что $k s \geqslant k_{1} s_{1}$, получаем

$$
T \leqslant 28 X Y d^{\frac{1}{2 k_{1} s_{1}}}(\lg m)^{-\frac{C}{16}}
$$

так что

$$
\left|W_{1}\right| \leqslant 56 X Y d^{\frac{1}{2 k_{1} s_{1}}}(\lg m)^{1-\frac{C}{16}} .
$$

Далее, из определения величины $u$ следует, что в сумме $W(X, Y)$ каждое значение $u$ повторяется не более

$$
(\mu-1) !(\nu-1) !=(\mu \nu)^{-1} \mu ! \nu !
$$

раз. Поэтому

$$
|W(X, Y)| \leqslant \sum_{u \leqslant x(X Y)^{-1}}^{\prime}(\mu \nu)^{-1} \mu ! \nu !\left|W_{1}\right|,
$$

где штрих в сумме по $u$ означает отсутствие повторяющихся слагаемых. Воспользовавшись найденной выше оценкой $W_{1}$, получим последовательно ряд неравенств:

$$
\begin{aligned}
& |W(X, Y)| \leqslant(\mu \nu)^{-1} \mu ! \nu ! x(X Y)^{-1} 56 X Y d^{\frac{1}{2 k_{1} s_{1}}}(\lg m)^{1-\frac{C}{16}}
\end{aligned}
$$

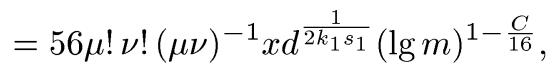

$$
\begin{aligned}
& (\mu ! \nu !)^{-1}|W| \leqslant(\mu ! \nu !)^{-1}(\lg m)^{3}(\mu \nu)^{-1} 56 \mu ! \nu ! x d^{\frac{1}{2 k_{1} s_{1}}}(\lg m)^{1-\frac{C}{16}} \\
& =56(\mu \nu)^{-1} x d^{\frac{1}{2 k_{1} s_{1}}}(\lg m)^{4-\frac{C}{16}}, \\
& \left|S_{\mu, \nu}\right| \leqslant(\mu ! \nu !)^{-1}|W|+\frac{2 x}{\lg x} \leqslant 56(\mu \nu)^{-1} x d^{\frac{1}{2 k_{1} s_{1}}}(\lg m)^{4-\frac{C}{16}}+\frac{2 x}{\lg x}, \\
& \sum_{\mu=1}^{\mu_{0}} \sum_{\nu=1}^{\nu_{0}}\left|S_{\mu, \nu}\right| \leqslant 2 \lg \mu_{0} \lg \nu_{0} \cdot 56 x d^{\frac{1}{2 k_{1} s_{1}}}(\lg m)^{4-\frac{C}{16}}+\frac{2 \mu_{0} \nu_{0} x}{\lg x} .
\end{aligned}
$$

Учитьвая неравенства (20), получим

$112 \lg \mu_{0} \lg \nu_{0}<\lg m$,

$$
\begin{aligned}
\frac{2 \mu_{0} \nu_{0} x}{\lg x} & <\frac{16 x}{(\lg x)^{2}} \lg m(\lg x)^{14 \gamma} \\
& =16 C^{-\frac{7}{12}} x(\lg m)^{-\frac{4}{3}}(\lg x)^{\frac{11}{12}}(\lg \lg m)^{-\frac{7}{12}}<x(\lg m)^{-\frac{1}{9}}
\end{aligned}
$$


и, наконец,

$$
\sum_{\mu=1}^{\mu_{0}} \sum_{\nu=1}^{\nu_{0}}\left|S_{\mu, \nu}\right|<x d^{\frac{1}{2 k_{1} s_{1}}}(\lg m)^{5-\frac{C}{16}}+x(\lg m)^{-\frac{1}{9}} .
$$

Если взять теперь $C=160$, то для $S_{1}$ получится следующая оценка:

$$
\begin{aligned}
\left|S_{1}\right| & \leqslant \sum_{\mu=0}^{\mu_{0}} \sum_{\nu=0}^{\nu_{0}}\left|S_{\mu, \nu}\right|<x \Delta+x d^{\frac{1}{2 k_{1} s_{1}}}(\lg m)^{-5}+x(\lg m)^{-\frac{1}{9}} \\
& \leqslant 2 x \Delta+x d^{\frac{1}{2 k_{1} s_{1}}}(\lg m)^{-5}=x\left(2 \Delta+\Delta_{1}\right) .
\end{aligned}
$$

Переходя к оценке $S$, будем иметь

$$
|S| \leqslant\left|S_{1}\right|+\frac{2 x}{\lg x}<x\left(2 \Delta+\Delta_{1}+\frac{2}{\lg x}\right)<x\left(3 \Delta+\Delta_{1}\right) .
$$

Поскольку

$3 \Delta=3 C^{\frac{1}{24}}(\lg x)^{-\frac{5}{24}}(\lg m)^{\frac{1}{6}}(\lg \lg m)^{\frac{1}{24}} \lg \lg x<4(\lg x)^{-\frac{5}{24}}(\lg m)^{\frac{1}{6}}(\lg \lg m)^{\frac{25}{24}}=\Delta_{2}$,

TO

$$
|S| \leqslant x\left(\Delta_{1}+\Delta_{2}\right)
$$

Теорема 1 доказана.

\section{§4. Дробные доли чисел вида $\frac{a n^{*}+b n}{m}$}

Оценка тригонометрической суммы, полученная в предыдущем параграфе, может быть использована в решении ряда вопросов, касающихся распределения дробных долей специального вида функций, когда аргумент пробегает "короткую” последовательность значений.

Пусть, как и вьше, $m \geqslant m_{1}$ - достаточно большое натуральное число, $x-$ произвольное число, удовлетворяющее неравенствам

$$
\exp \left((\lg m)^{\frac{4}{5}}(\lg \lg m)^{\frac{73}{5}}\right) \leqslant x \leqslant m^{\frac{4}{7}}
$$

$a, b$ - целье числа, причем $(a, m)=1$. Обозначим $N$ количество чисел, не превосходящих $x$ и взаимно простых с $m$.

Ниже будут доказаны две следующие теоремы.

ТЕОРема 2. Пусть $\alpha и \beta$ - вещественные числа, подчиненные условию $0 \leqslant \alpha<\beta<1, K(\alpha, \beta)$ - число решений системы неравенств

$$
\alpha \leqslant\left\{\frac{a n^{*}+b n}{m}\right\}<\beta, \quad 1 \leqslant n \leqslant x, \quad(n, m)=1 .
$$

Тогда имеет место асимптотическая формула

$$
K(\alpha, \beta)=(\beta-\alpha) N+O(x \Delta)
$$

$2 \partial e$

$$
\Delta=(\lg x)^{-\frac{5}{24}}(\lg m)^{\frac{1}{6}}(\lg \lg m)^{\frac{49}{24}}
$$

а постоянная в знаке $O$ абсолютная. 
Теорема 3. Справедлива асимптотическая формула

$$
\sum_{n \leqslant x}^{\prime}\left\{\frac{a n^{*}+b n}{m}\right\}=\frac{1}{2} N+O(x \Delta)
$$

əде

$$
\Delta=(\lg x)^{-\frac{5}{24}}(\lg m)^{\frac{1}{6}}(\lg \lg m)^{\frac{49}{24}},
$$

а постоянная в знаке $O$ абсолютная.

Прежде чем приступить к доказательству этих утверждений, отметим два обстоятельства. Во-первых, задавшись некоторым $0<\delta<\frac{1}{16}$ и целым $\rho \geqslant 1$, утверждение теоремы 2 достаточно доказать для случая $2 \delta<\beta-\alpha<1-2 \delta$, поскольку при $0<\beta-\alpha \leqslant 2 \delta$

$$
K(\alpha, \beta)=K(\alpha, \alpha+1-2 \delta)-K(\beta, \alpha+1-2 \delta),
$$

а при $1-2 \delta \leqslant \beta-\alpha<1$

$$
K(\alpha, \beta)=K\left(\alpha, \alpha+\frac{1}{2}\right)+K\left(\alpha+\frac{1}{2}, \beta\right) .
$$

Во-вторых, из леммы 7 следует, что обе теоремы будут доказаны (первая - для случая $2 \delta<\beta-\alpha<1-2 \delta$ ), если будет получена соответствующая асимптотика для суммы

$$
U(\alpha, \beta)=\sum_{n \leqslant x} \psi\left(\frac{a n^{*}+b n}{m}\right),
$$

где $\psi(x)$ - “стаканчик" Виноградова, построенный по заданным $\delta, \rho, \alpha$ и $\beta$ (лемма 6). Поэтому мы объединим доказательства обоих утверждений.

ДокАЗАТЕЛЬСТво. Возьмем $r_{0}=(\lg m)^{36}, \delta=r_{0}^{-1 / 2}, \rho=\left[r_{0}^{1 / 4}\right]$ и будем считать, что $2 \delta<\beta-\alpha<1-2 \delta$. Пользуясь леммой 6 , построим "стаканчик" Виноградова $\psi(x)$, отвечаюший выбранньм $\delta, \rho, \alpha$ и $\beta$, и рассмотрим сумму $U(\alpha, \beta)$. Разлагая $\psi$ в ряд Фурье, получим для $U(\alpha, \beta)$ выражение

$$
U(\alpha, \beta)=(\beta-\alpha) N+R,
$$

где

$$
R=\sum_{r \neq 0} g(r) S_{r}, \quad S_{r}=\sum_{n \leqslant x}^{\prime} \exp \left(2 \pi i r \frac{a n^{*}+b n}{m}\right) .
$$

Оценим величину $R$. Прежде всего заметим, что

$$
R \ll \sum_{r=1}^{+\infty} c(r)\left|S_{r}\right|,
$$

где величины $c(r)$ определены в (12). Далее, сумму по $r$ разобьем на две, так что

$$
R \ll\left(\sum_{r \leqslant r_{0}}+\sum_{r>r_{0}}\right) c(r)\left|S_{r}\right|=R_{1}+R_{2},
$$


и оценим каждую из этих сумм по-своему. Если $r \leqslant r_{0}$, то $d=(a r, m) \leqslant r \leqslant r_{0}$. Оценивая $S_{r}$ с помошью теоремы 1 , получим

$$
\left|S_{r}\right| \leqslant x\left(\Delta_{1}+\Delta_{2}\right)
$$

причем

$$
\begin{aligned}
& \Delta_{1}=r_{0}^{\frac{1}{2 k_{1} s_{1}}}(\lg m)^{-5} \leqslant r_{0}^{\frac{1}{18}}(\lg m)^{-5}=(\lg m)^{-3}, \\
& \Delta_{2}=4(\lg x)^{-\frac{5}{24}}(\lg m)^{\frac{1}{6}}(\lg \lg m)^{\frac{25}{24}} .
\end{aligned}
$$

Поскольку $\Delta_{1}<\Delta_{2}$, то $\left|S_{r}\right| \ll x \Delta_{2}$. Поэтому

$$
R_{1} \ll \sum_{r \leqslant r_{0}} c(r) x \Delta_{2} \ll x \Delta_{2} \sum_{r \leqslant r_{0}} \frac{1}{r} \ll x \Delta_{2} \lg r_{0} \ll x \Delta_{2} \lg \lg m \ll x \Delta,
$$

где $\Delta=(\lg x)^{-\frac{5}{24}}(\lg m)^{\frac{1}{6}}(\lg \lg m)^{\frac{49}{24}}$.

Сумму $R_{2}$ оценим тривиально:

$$
R_{2} \leqslant \sum_{r>r_{0}} \frac{1}{\pi r}\left(\frac{\rho}{r \delta}\right)^{\rho} x \ll \frac{x}{r_{0}}\left(\frac{\rho}{r_{0} \delta}\right)^{\rho} \ll \frac{x}{r_{0}} r_{0}^{-\frac{\rho}{4}} \ll x \Delta .
$$

Таким образом, $R \ll x \Delta$, и

$$
U(\alpha, \beta)=(\beta-\alpha) N+O(x \Delta) .
$$

Пользуясь леммой 7 и замечая, что

$$
N \delta \ll x(\lg m)^{-18} \ll x \Delta,
$$

получаем утверждения теорем 2 и 3 .

В заключение выясним, когда асимптотические формулы для величин $K(\alpha, \beta)$ и $S=\sum_{n \leqslant x}^{\prime}\left\{\frac{a n^{*}+b n}{m}\right\}$ нетривиальны, т.е. когда остаточный член будет меньше главного. Для этого необходимо оценить величину $N$. Имеем

$$
\begin{aligned}
N & =\sum_{n \leqslant x}^{\prime} 1=\sum_{n \leqslant x} \sum_{d \mid(n, m)} \mu(d)=\sum_{\substack{d \mid m \\
d \leqslant x}} \mu(d)\left[\frac{x}{d}\right]=\sum_{d \mid m} \mu(d)\left[\frac{x}{d}\right] \\
& =x \sum_{d \mid m} \frac{\mu(d)}{d}-\sum_{d \mid m} \mu(d)\left\{\frac{x}{d}\right\}=\frac{\varphi(m)}{m} x+\theta \cdot \tau(m),
\end{aligned}
$$

где $|\theta| \leqslant 1$. Далее, как известно,

$$
c_{1}(\lg \lg m)^{-1} \leqslant \frac{\varphi(m)}{m}<1
$$

где $c_{1}>0$ - абсолютная постоянная. Поэтому

$$
N \geqslant \frac{c_{1} x}{\lg \lg m}-\tau(m) .
$$


Пусть $m$ таково, что $\tau(m)$ мало, т.е. для $\tau(m)$ выполнено неравенство

$$
\tau(m) \leqslant \frac{c_{1} x}{2 \lg \lg m}
$$

(это выполнено, например, когда $m=p$ - простое число). Тогда

$$
N \geqslant \frac{c_{1} x}{2 \lg \lg m} .
$$

Асимптотическая формула для $S$ будет нетривиальной, если

$$
x \Delta \ll x(\lg \lg m)^{-1},
$$

т.е. если выполнено неравенство

$$
\lg x \gg(\lg m)^{\frac{4}{5}}(\lg \lg m)^{\frac{73}{5}} .
$$

При выполнении этого условия будет нетривиальной и асимптотика для величины $K(\alpha, \beta)$, если только $\alpha$ и $\beta$ - фиксированные числа. В обшем же случае ввиду соотношения

$$
\varlimsup_{m \rightarrow \infty} \lg \tau(m) \frac{\lg \lg m}{\lg m}=\lg 2
$$

асимптотические формулы для $S$ и $K(\alpha, \beta)$ будут нетривиальны, вообще говоря, лишь при

$$
x \geqslant \exp \left(c_{2} \frac{\lg m}{\lg \lg m}\right)
$$

где $c_{2}>\lg 2$.

\section{Список литературы}

1. Kloosterman H. D. On the representation of numbers in the form $a x^{2}+b y^{2}+c z^{2}+d t^{2}$ // Acta Math. 1926. V. 49. P. 407-464.

2. Salie H. Über die Kloostermanschen Summen $S(u, v, q) / /$ Math. Z. 1931. T. 34. S. 91-109.

3. Weil A. On some exponential sums // Proc. Nat. Acad. Sci. USA. 1948. V. 34. P. 204-207.

4. Carlitz L., Uchiyama S. Bounds for exponential sums // Duke Math. J. 1957. V. 24. P. $37-41$.

5. Карацуба А. А. Распределение обратных величин в кольце вычетов по заданному модулю // Докл. РАН. 1993. Т. 333. №2. С. 138-139.

6. Карацуба А. А. Дробные доли специального вида функций // Изв. РАН. Сер. матем. 1995. T. 59. № 4. C. $61-80$.

7. Карачуба А. А. Аналоги сумм Клоостермана // Изв. РАН. Сер. матем. 1995. Т. 59. №5. C. $93-102$.

8. Караиуба А. А. Суммы дробных долей специального вида функций // Докл. РАН. 1996. T. 349. № 3. C. 302.

9. Карацуба А. А. Аналоги неполных сумм Клоостермана и их приложения // Tatra Mountains Math. Publ. 1997. V. 11. P. 89-120.

10. Прахар К. Распределение простых чисел. М.: Мир, 1967.

11. Виноградов И. М. Метод тригонометрических сумм в теории чисел. 2-е изд. М.: Наука, 1983. 